\title{
Relapsing peritonitis with Bacillus cereus in a patient on continuous ambulatory peritoneal dialysis
}

\author{
Eyð Tausen Magnussen, ${ }^{1}$ Amanda Gratton Vang, ${ }^{2}$ Torkil á Steig, ${ }^{1}$ Shahin Gaini $3,4,5$
}

\begin{abstract}
${ }^{1}$ Medical Department, Nationa Hospital Faroe Islands, Tórshavn, Faroe Islands ${ }^{2}$ Department of Diagnostic Medicine, National Hospital Faroe Islands, Tórshavn, Faroe Islands

${ }^{3}$ Medical Department, Infectious Diseases Division, National Hospital Faroe Islands, Tórshavn, Faroe Islands

${ }^{4}$ Department Infectious Diseases, Odense University Hospital, Odense, Denmark ${ }^{5}$ Faculty of Science and Technology, University of the Faroe Islands, Tórshavn, Faroe Islands
\end{abstract}

\section{Correspondence to} Dr Shahin Gaini, shahingaini@hotmail.com

Accepted 12 April 2016

\section{CrossMark}

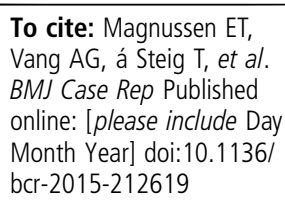

\section{SUMMARY}

We present a case where Bacillus cereus was determined to be the causative agent of relapsing peritonitis in a patient on continuous ambulatory peritoneal dialysis (CAPD). The patient, a 70-year-old man from the Faroe Islands, was admitted with relapsing peritonitis four times over a 3-month period. Peritoneal cultures were positive for growth of $B$. cereus, a rare bacterial cause of peritonitis. The cultures demonstrated susceptibility to vancomycin, and therefore the patient was treated with intraperitoneal vancomycin, intraperitoneal gentamycin and oral ciprofloxacin. As a result of the relapsing $B$. cereus peritonitis diagnosis and a CT scan showing contraction of the peritoneum after longstanding inflammation, the peritoneal catheter was removed and the patient converted to haemodialysis. To date, the patient has not been readmitted due to peritonitis. A lack of proper hygiene when changing the dialysis bag was the suspected source of infection with $B$. cereus.

\section{BACKGROUND}

Peritonitis is the most common complication associated with peritoneal dialysis. ${ }^{1}$ However, only nine cases of peritonitis with Bacillus cereus in patients on peritoneal dialysis have been reported in the literature. $^{2-9}$ In this report, we use peritoneal dialysis to refer to patients who have received continuous ambulatory peritoneal dialysis (CAPD), intermittent peritoneal dialysis as well as automated peritoneal dialysis. Our new report from the Faroe Islands adds to the critical knowledge about this rare infection, and shares clinically useful information regarding treatment and potential sources of infection.

\section{CASE PRESENTATION}

A 70-year-old Caucasian man on CAPD since 2010 was hospitalised on 21 September 2012, reporting of abdominal pain. The patient had known heart disease and was on CAPD due to terminal kidney disease caused by membranous glomerulonephritis, which was diagnosed in 2005. The patient had a history of hospitalisation due to peritonitis (table 1).

It is important to note that the patient refused to have his catheter removed at an early stage of his CAPD treatment and insisted on CAPD as renal replacement therapy. The peritoneal catheter in place at his 2012 hospital admission was the original catheter from 2010 and he had never been on haemodialysis. The procedure he routinely used with his CAPD was: four exchanges a day using Gambrosol, TRIO three times daily and Extraneal (Baxter) overnight. Inflow volume was $2 \mathrm{~L}$ per bag.
Peritonitis was suspected at this admission as well. Peritoneal fluid was cultured and empirical antibiotics vancomycin (2 g, 1 dose) and Gentamycin (80 mg, 1 dose) were administered intraperitoneally. The patient's dry body weight was 98 kgs. Results from the peritoneal fluid culture showed growth of $B$. cereus, a Gram-positive rod, susceptible to vancomycin. The patient reported that, preceding symptom onset, he had handled two plants received as a gift and then proceeded to change his dialysis bag. It is possible that this lack of proper hygiene was responsible for the infection with the atypical pathogen B. cereus.

The empirical antibiotic regimen successfully relieved the abdominal pain and the patient was in good condition. Therefore, it was decided to treat him on an outpatient basis. On discharge, the patient received gentamycin $(80 \mathrm{mg}$, once daily for 1 week) and vancomycin (2 g, 1 dose administered 1 week after discharge) to be administered intraperitoneally. In addition, oral treatment with ciprofloxacin (500 mg, twice daily for 10 days) was prescribed as prophylaxis against Gram-negative bacteria.

On 3 November 2012, the patient was rehospitalised with abdominal pain and fever. Peritoneal fluid was again examined and cultured. The peritoneal fluid was turbid and cloudy; taken together with the patient's history, peritonitis was again suspected. The patient was then treated with vancomycin and gentamycin intraperitoneally as in the previous hospitalisation. Treatment with antibiotics again caused the abdominal pain to resolve. Results of the peritoneal fluid culture showed a recurrence of $B$. cereus with the same antibiotic susceptibility found previously. Gentamycin was discontinued and vancomycin prescribed intraperitoneally once a week for 4 weeks in total.

Five days later, on 8 November 2012, the patient presented with increased abdominal pain, fever and reduced appetite. He was readmitted to the hospital for the third time since September 2012. The peritoneal fluid was clear this time. However, $B$. cereus peritonitis was again identified by culturing. The patient was treated with vancomycin $(2 \mathrm{~g}$, once a week for 4 weeks) and gentamycin $(80 \mathrm{mg}$, once daily for 6 days) intraperitoneally. Oral treatment with ciprofloxacin $(500 \mathrm{mg}$, twice daily for 10 days) was repeated. Unlike the two previous episodes of peritonitis, the abdominal pain persisted and a CT scan showed displacement of the peritoneal catheter, with neither abscess formation nor intestinal perforation. Displacement of the dialysis catheter was from the fossa rectovesicalis to the 
Table 1 Peritonitis episodes prior to September 2012

\begin{tabular}{ll}
\hline Date & Bacterial aetiology \\
\hline 19 May 2010 & Enterococcus faecalis \\
3 June 2010 & $*$ \\
19 July 2010 & Pseudomonas oryzihabitans \\
& Enterococcus faecalis \\
15 February 2011 & Enterococcus faecalis \\
21 February 2011 & Enterococcus faecalis \\
19 March 2011 & No growth \\
1 June 2011 & Enterobacter cloacae \\
22 August 2011 & Gram-positive rods* \\
7 October 2011 & Enterococcus faecalis \\
26 November 2011 & Enterococcus faecalis \\
9 February 2012 & Gram-positive rods* \\
8 March 2012 & Klebsiella pneumonia \\
5 April 2012 & Staphylococcus epidermidis \\
8 June 2012 & Pseudomonas fluorescens \\
11 July 2012 & Staphylococcus epidermidis \\
\hline *Unavailable. &
\end{tabular}

space in front of the bladder just behind the symphysis pubica. The catheter was determined to still be functional and an attempt was made to replace the catheter under X-ray guidance, but failed. Since the catheter still functioned and the abdominal pain was relieved, the patient was discharged after 6 days.

On 29 December 2012, a new episode of peritonitis with $B$. cereus was diagnosed for this patient. A new CT scan with contrast injected through the peritoneal catheter showed inflammation along the catheter and contrast accumulation in a limited cavity in the right abdominal cavity (figures 1 and 2). It was not possible to continue peritoneal dialysis under these circumstances. Therefore, the patient was sent to a tertiary centre where the dialysis catheter was removed and the patient converted to haemodialysis. A cultured sample taken from the exit site showed growth of Staphylococcus haemolyticum. Unfortunately, no sample was taken from the catheter itself when it was removed.

Since the cluster of B. cereus-positive peritonitis at the end of 2012, the patient has not been hospitalised again for peritonitis. He has, however, been hospitalised twice since 2012 for issues

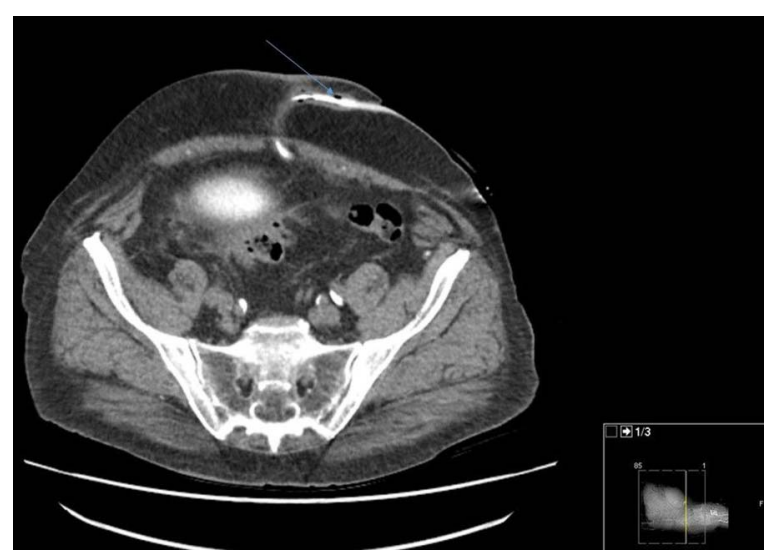

Figure 1 Abdominal CT (axial slice) with contrast injected through the peritoneal dialysis catheter with the arrow indicating inflammatory changes subcutaneously along the peritoneal catheter and air bubbles along the catheter also indicating inflammation.

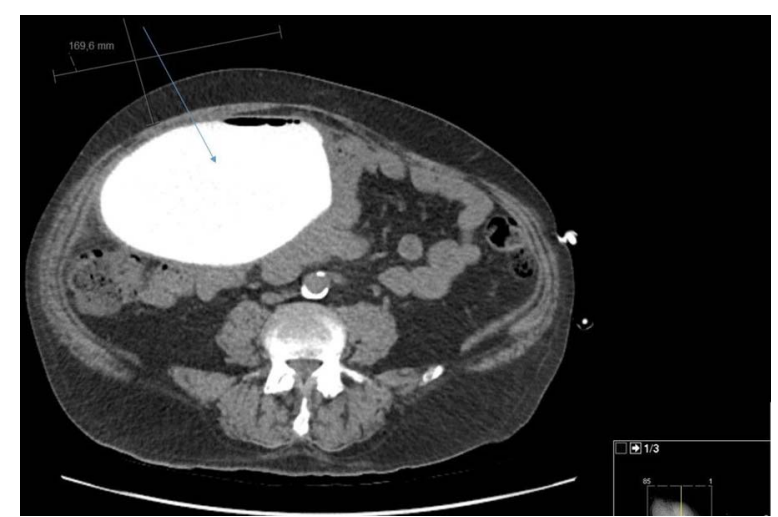

Figure 2 Abdominal CT (axial slice) showing accumulation of contrast fluid in a limited $10 \times 14 \times 17 \mathrm{~cm}$ cavity in the right abdominal cavity, marked with an arrow, caused by inflammatory contraction of the peritoneum after longstanding inflammation.

unrelated to the peritonitis-once because of pneumonia and once because of a secondary skin infection related to itching and high plasma phosphate levels.

\section{DISCUSSION}

Peritonitis caused by $B$. cereus is very rare. There are 13 previously reported cases of $B$. cereus peritonitis. In 2 of the 13 cases, $B$. cereus caused peritonitis after caesarean section in otherwise healthy women. ${ }^{10}$ Patients in 2 of the 13 cases had chronic liver disease and developed spontaneous $B$. cereus peritonitis. ${ }^{11} 12$ The remaining nine cases were patients on peritoneal dialysis (table 2). B. cereus caused relapsing peritonitis in six cases on peritoneal dialysis. All six patients with CAPD presented with abdominal pain. The discovery of $B$. cereus resulted in replacement of the dialysis catheter in three of the six reports and conversion to haemodialysis in one of the cases. ${ }^{569}$ One of the cases was a paediatric patient who underwent a kidney transplantation, while the other three cases responded well to empirical antibiotic treatment. ${ }^{2} 378$

$B$. cereus is a Gram-positive endospore forming rod of which the natural reservoir is rotten organic material, salt water and fresh water, vegetables and the bowels of invertebrates. ${ }^{13}$ It produces $\beta$ lactamases and is therefore often resistant to $\beta$-lactam antibiotics. ${ }^{13}$ The most frequent source of infection is food and soil. ${ }^{13}$ B. cereus causes food poisoning through rice, milk and pasta that are not correctly prepared or not heated properly. ${ }^{7} 13$ The spores are resistant to cold, heat, radiation and desiccation. ${ }^{3} 13$ Besides gastrointestinal symptoms, B. cereus has in some cases been shown to cause more severe conditions such as central nervous system infections, sepsis, severe pneumonia, panophthalmitis and severe skin infections. Severe infections are more common in immunocompromised patients, patients with implanted medical equipment and intravenous drug abusers. $^{5} 12 \quad 13$ B. cereus has hydrophobic properties, which facilitate adherence to different surfaces, such as catheters. B. cereus also produces biofilm, a matrix of polysaccharide, which surrounds bacteria and drastically reduces the ability of antibiotics to penetrate.

In the case we report here, as well as in four other cases, there was suspicion of contamination from the surrounding environment. $^{2}{ }^{5}$ In one of these studies, ${ }^{5}$ of a $B$. cereus infected patient who lived on a farm, the authors hypothesised that soil and dust could have been contributory factors to the infection. In another study, ${ }^{3}$ bad hygiene in the home was 
Table 2 Overview of case reports describing Bacillus cereus peritonitis in patients on peritoneal dialysis

\begin{tabular}{|c|c|c|c|c|}
\hline Sex & Age & Treatment & Outcome & References \\
\hline M & 73 & IV vancomycin+IV netilmicin & Complete cure & 2 \\
\hline $\mathrm{F}$ & 11 & $\begin{array}{l}\text { 1. IP vancomycin+IP ceftazidime } \\
\text { 2. IP cephalothin+IP ceftazidime+PO ciprofloxacin+PO nystatin }\end{array}$ & Relapse, change of antibiotic treatment, then complete cure & 3 \\
\hline M & 71 & $\begin{array}{l}\text { 1. IP cefotaxime } \\
\text { 2. IP gentamicin }\end{array}$ & Relapse, then death after cardiac arrest & 4 \\
\hline $\mathrm{F}$ & 63 & $\begin{array}{l}\text { 1. IP gentamicin+IP vancomycin } \\
\text { 2. IP vancomycin } \\
\text { 3. IV vancomycin }\end{array}$ & Relapse twice, catheter removed, then complete cure & 5 \\
\hline M & 69 & $\begin{array}{l}\text { 1. IP cefuroxime+IP ceftazidime } \\
\text { 2. IP tobramycin } \\
\text { 3. IP vancomycin+IV ciprofloxacin }\end{array}$ & Relapse, converted to haemodialysis & 6 \\
\hline M & 65 & IV vancomycin+IV gentamicin & Complete cure & 7 \\
\hline M & 67 & IP teicoplanin+IP aztreonam & Complete cure & 8 \\
\hline $\mathrm{F}$ & 60 & $\begin{array}{l}\text { 1. IP gentamicin+cefuroxim } \\
\text { 2. IP gentamicin+IP vancomycin } \\
\text { 3. PO cotrimoxazole } \\
\text { 4. Vancomycin for } 6 \text { weeks* }\end{array}$ & Relapse twice, catheter removed, then complete cure & Case $1^{9}$ \\
\hline $\mathrm{F}$ & 62 & $\begin{array}{l}\text { 1. IP gentamicin+IP cefuroxim } \\
\text { 2. IP vancomycin } \\
\text { 3. PO ciprofloxacin }\end{array}$ & Relapse twice, catheter removed, then complete cure & Case $2^{9}$ \\
\hline
\end{tabular}

reported, potentially leading to colonisation of the catheter. In a third study, ${ }^{2}$ the patient admitted he had not used correct changing techniques and may have touched the catheter connector. In a further study, ${ }^{8}$ the patient admitted he did not wash his hands after preparing a 'roll-up' cigarette before changing the dialysis bag.

In the case we report here, the patient indicated that he had handled plants he had received as a gift and changed the dialysis bag shortly thereafter. The precedent from the cases reported in the literature suggests that contamination from the soil, combined with suboptimal hygiene when changing the dialysis bag, could be responsible for the $B$. cereus peritonitis in our case as well. No data are available to prove if $B$. cereus exists naturally in Faroese soil. All plants for indoor use in the Faroe Islands are imported from abroad, and there is no local Faroese production of houseplants.

\section{Learning points}

- In most cases, identification of Bacillus cereus is considered to be a sampling contamination because of its wide distribution in the environment. However, as seen in the case above, it can cause opportunistic infections.

- Production of biofilm by $B$. cereus makes antibiotic treatment of peritonitis challenging. In many cases, treatment success requires changing the dialysis catheter.

- The importance of proper hygiene when changing the dialysis catheter and maintaining an acceptable level of hygiene in the patient's environment should be discussed with the patient to avoid contaminating the dialysis catheter during routine use.

- Clinicians treating patients on peritoneal dialysis should be aware of the possibility that $B$. cereus can cause peritonitis in patients on peritoneal dialysis. In addition, clinicians should be especially aware of contamination from soil originating from houseplants.
Acknowledgements The authors would like to thank Dr Marius Rasmussen, radiologist, for his help interpreting the CT scan used for the figures in the report.

Contributors ETM was involved in planning and was the lead author of the manuscript. SG involved in planning and critical revision. Tás was involved in treating the patient and contributing details to the manuscript. AGV researcher: proofreading and contributing to revision of the manuscript.

Competing interests None declared.

Patient consent Obtained.

Provenance and peer review Not commissioned; externally peer reviewed.

\section{REFERENCES}

1 Stuart S, Booth TC, Cash CJC, et al. Complications of continuous ambulatory peritoneal dialysis. Radiographics 2009;29:441-60.

2 Balakrishnan I, Baillod RA, Kibbler CC, et al. Bacillus cereus peritonitis in a patient being treated with continuous ambulatory peritoneal dialysis. Nephrol Dial Transplant 1997:12:2447-8.

3 Monteverde ML, Sojo ET, Grosnan M, et al. Realpsing Bacillus cereus peritonitis in a pediatric patient on chronic peritoneal dialysis. Perit Dial Int 2006;26:715-16.

4 Biasioli S, Chiaramonte $S$, Fabris $A$, et al. Bacillus cereus as agent of peritonitis during peritoneal dialysis. Nephron 1984;37:211-12.

5 Ros Ruiz S, Martin Reyes G, Trujillo Campos C, et al. Bacillus cereus peritonitis during automated peritoneal dialysis. Perit Dial Int 2006;26:612-13.

6 Spiliopoulou A, Papachristou E, Foka A, et al. Relapsing Bacillus cereus peritonitis in a patient treated with continuous ambulatory peritoneal dialysis. JMM Case Repo 2014.

7 Ai-Hilali N, Nampoory MRN, Johny KV, et al. Bacillus cereus peritonitis in a chronic peritoneal dialysis patient. Perit Dial Int 1997;17:514-15.

8 al-Wali W, Baillod R, Hamilton-Miller JM, et al. Detective work in continuous ambulatory peritoneal dialysis. J Infect 1990;20:151-4.

9 Pinedo S, Bos AJ, Siegert CEH. Relapsing Bacillus cereus Peritonitis in Two patients on peritoneal dialysis. Perit Dial Int 2002;22:424-6.

10 Shimoni $Z$, Namet $Y$, Niven $M$, et al. Bacillus cereus peritonitis after Cesarean section. Surg Infect (Larchmt) 2008;9:105-6.

11 Lee YL, Shih SD, Weng YJ, et al. Fatal spontaneous bacterial peritonitis and necrotizing fascitis with bacteraemia caused by Bacillus cereus in a patient with cirrhosis. J Med Microbiol 2010;59:242-4.

12 Bhattacharyya S, Ansari MAA, Sartaz A, et al. Spontaneous Bacterial Peritonitis (SBP) caused by Bacillus cereus in an Alcoholic patient: case report and review of literature. J Clin Diagn Res 2015;9:DD03-4.

13 Bottone EJ. Bacillus cereus, a volatile human pathogen. Clin Microbio/ Rev 2010;23:382-98. 
Copyright 2016 BMJ Publishing Group. All rights reserved. For permission to reuse any of this content visit http://group.bmj.com/group/rights-licensing/permissions.

BMJ Case Report Fellows may re-use this article for personal use and teaching without any further permission.

Become a Fellow of BMJ Case Reports today and you can:

- Submit as many cases as you like

- Enjoy fast sympathetic peer review and rapid publication of accepted articles

- Access all the published articles

- Re-use any of the published material for personal use and teaching without further permission

For information on Institutional Fellowships contact consortiasales@bmjgroup.com

Visit casereports.bmj.com for more articles like this and to become a Fellow 